\title{
Do Anesthetic Techniques Influence the Threshold for Glomerular Capillary Hemorrhage Induced in Rats by Contrast- Enhanced Diagnostic Ultrasound?
}

\author{
Douglas L. Miller, PhD, Xiaofang Lu, PhD, Mario Fabiilli, PhD, Chunyan Dou, MD
}

Received May 6, 2015, from the Department of Radiology, University of Michigan Health System, Ann Arbor, Michigan USA. Manuscript accepted for publication June 6, 2015.

This work was supported by Public Health Service grant HL110990, awarded by the National Institutes of Health, US Department of Health and Human Services.

Address correspondence to Douglas L. Miller, PhD, University of Michigan Health System, 3240A Medical Science Building I, 1301 Catherine St, Ann Arbor, MI 48109-5667 USA.

E-mail:douglm@umich.edu

Abbreviations

PRPA, peak rarefactional pressure amplitude; US, ultrasound

doi:10.7863/ultra.15.05015
Objectives - Glomerular capillary hemorrhage can be induced by ultrasonic cavitation during contrast-enhanced diagnostic ultrasound (US) exposure, an important nonthermal US bioeffect. Recent studies of pulmonary US exposure have shown that thresholds for another nonthermal bioeffect of US, pulmonary capillary hemorrhage, is strongly influenced by whether xylazine is included in the specific anesthetic technique. The objective of this study was to determine the influence of xylazine on contrastenhanced diagnostic US-induced glomerular capillary hemorrhage.

Methods - In this study, anesthesia with ketamine only was compared to ketamine plus xylazine for induction of glomerular capillary hemorrhage in rats by $1.6-\mathrm{MHz}$ intermittent diagnostic US with a microsphere contrast agent (similar to Definity; Lantheus Medical Imaging, Inc, North Billerica, MA). Glomerular capillary hemorrhage was measured as a percentage of glomeruli with hemorrhage found in histologic sections for groups of rats scanned at different peak rarefactional pressure amplitudes.

Results - There was a significant difference between the magnitude of the glomerular capillary hemorrhage between the anesthetics at $2.3 \mathrm{MPa}$, with $45.6 \%$ hemorrhage for ketamine only, increasing to $63.2 \%$ hemorrhage for ketamine plus xylazine $(P<.001)$. However, the thresholds for the two anesthetic methods were virtually identical at 1.0 $\mathrm{MPa}$, based on linear regression of the exposure response data.

Conclusions - Thresholds for contrast-enhanced diagnostic US-induced injury of the microvasculature appear to be minimally affected by anesthetic methods.

Key Words - contrast-enhanced diagnostic ultrasound; diagnostic ultrasound adverse effects; glomerular hemorrhage; ultrasonic cavitation biology

7 he kidney is an excellent organ for ultrasound (US) examination, owing to its shallow depth and minimal obstruction by ribs. Contrast agents developed for diagnostic US are injectable suspensions of gas bodies (stabilized microbubbles), which provide a strong echo return for image enhancement. ${ }^{1}$ Contrast-enhanced diagnostic US works well in the kidney and rivals magnetic resonance imaging or computed tomography for diagnostic imaging. ${ }^{2}$ The method may be especially useful in patients for whom magnetic resonance imaging or computed tomography contrast is contraindicated ${ }^{3}$ and in children for ionizing dose minimization. ${ }^{4}$ Renal contrast-enhanced diagnostic US is recommended for suspected vascular disorders and for differential diagnosis between solid lesions and cysts with an equivocal appearance on conventional US imaging. 5 
Contrast agent gas bodies can be destabilized at peak rarefactional pressure amplitudes (PRPAs) within the diagnostic range. Under some conditions, this destabilization can lead to nucleation of acoustic cavitation, a wellknown mechanism for biological effects of US. Research into potential bioeffects of contrast-enhanced diagnostic US has identified several bioeffects, particularly during intermittent modes of imaging, which use gas body destabilization and destruction for imaging of perfusion on refill. ${ }^{6}$ In the kidney, intermittent contrast-enhanced diagnostic US at a high mechanical index has been shown to induce glomerular capillary hemorrhage into the urinary space, with hematuria and visible petechiae on the kidney surface. ${ }^{7,8}$ This effect increased rapidly above a threshold, which depended on the agent dose, exposure duration, and intermittent frame interval, with no effect seen for continuous imaging. The effect has been shown to occur in a porcine model of human contrast-enhanced diagnostic US. ${ }^{9}$ The PRPA thresholds were found to be approximately proportional to the US frequency. ${ }^{10}$ Glomerular capillary hemorrhage induced by contrast-enhanced diagnostic US affects the associated nephron tubular system. Changes in affected regions were indicative of intratubular obstruction, leading to localized acute tubular necrosis, ${ }^{11}$ and interstitial fibrosis was evident after 4 weeks. ${ }^{12}$ However, the clinical occurrence and medical importance of contrastenhanced diagnostic US-induced glomerular capillary hemorrhage remain uncertain, and research is needed on factors that could help define the possible clinical occurrence of the effects seen in laboratory research.

Another nonthermal bioeffect associated with diagnostic US scanning is pulmonary capillary hemorrhage. Discovered over 25 years ago, ${ }^{13}$ this effect has received substantial interest as a safety issue. ${ }^{14}$ Recent research has revealed that the anesthetic methods used to enable US scanning in rats have an important role in the magnitude and threshold of pulmonary capillary hemorrhage. ${ }^{15,16}$ Ketamine plus xylazine has been used extensively in pulmonary capillary hemorrhage research, and xylazine can affect pulmonary physiologic characteristics, inducing edema at relatively high doses. ${ }^{17,18}$ Miller et al ${ }^{16}$ examined the influence of other anesthetic techniques, including ketamine only, pentobarbital, inhalational isoflurane, and the supplemental use of xylazine with ketamine or isoflurane. A diagnostic US machine with a 7.6-MHz linear array was used to image the right lung of anesthetized rats in a warmed water bath, and pulmonary capillary hemorrhage was measured on the lung surface. The hemorrhage was greatest for pentobarbital, lower for inhalational isoflurane, and lowest for ketamine-only anesthesia. Addition of xylazine pro- duced a substantial increase in hemorrhage. Thresholds for pulmonary capillary hemorrhage were lower by nearly a factor of 2 for ketamine plus xylazine or pentobarbital than thresholds for anesthesia with ketamine only or isoflurane only. These results indicate a role for experimental anesthetic methods in pulmonary capillary hemorrhage occurrence and complicate the interpretation of results for clinical US examinations.

Many studies of bioeffects in contrast-enhanced diagnostic US have also used ketamine plus xylazine for anesthesia because it is the recommended anesthetic method for rats. This method is favored because of the depth of anesthesia and analgesia for surgical procedures and the long duration (typically longer than 1 hour) of stable anesthesia. Given the effects on the thresholds and magnitude of pulmonary capillary hemorrhage in the research noted above, the question arises as to whether the use of xylazine in the anesthetic method might also affect the results of research on microvascular bioeffects associated with contrastenhanced diagnostic US. In particular, xylazine is known to affect kidney function. Xylazine acts as a diuretic, ${ }^{19,20}$ which is quite apparent when using ketamine plus xylazine anesthesia in research on rats. This factor was noted in previous research on contrast-enhanced diagnostic USinduced glomerular capillary hemorrhage, for which ketamine plus xylazine was tested in comparison to pentobarbital anesthesia. ${ }^{8}$ Pentobarbital does not induce apparent diuresis, but no significant difference in glomerular capillary hemorrhage was found for pentobarbital compared to ketamine plus xylazine anesthesia. However, the pulmonary US research also showed that pulmonary capillary hemorrhage thresholds were not different for pentobarbital compared to ketamine plus xylazine anesthesia. Thus, the question of anesthetic influence on thresholds for the glomerular capillary hemorrhage bioeffect remains unsettled. The objective of this study was to determine the influence of xylazine on contrast-enhanced diagnostic USinduced glomerular capillary hemorrhage using methods similar to the studies of pulmonary capillary hemorrhage, which compared anesthesia with ketamine plus xylazine to ketamine only. ${ }^{15,16}$

\section{Materials and Methods}

\section{Animal Preparation}

This in vivo research was conducted with the approval of the University Committee on the Use and Care of Animals at the University of Michigan. A total of 41 rats (CD hairless; Charles River Laboratories, Wilmington, MA) were used in this study. The rats were anesthetized via intraperitoneal 
injection of either ketamine $(91 \mathrm{mg} / \mathrm{kg})$ plus xylazine $(9 \mathrm{mg} / \mathrm{kg})$ or ketamine only $(100 \mathrm{ml} / \mathrm{kg})$. For intravenous injections and infusion of the contrast agent, a 24-gauge cannula was inserted into a tail vein. The anesthetized rats were mounted on a plastic holder with the left side toward the board. This mount was then placed vertically in a water bath filled with $38^{\circ} \mathrm{C}$ degassed water for scanning of the right side. Shaving was not needed for preparation because the rats were nearly hairless.

\section{Diagnostic US}

The general procedure for diagnostic US exposure with a contrast agent has been described previously. ${ }^{8}$ A phased array probe (S3, Vivid 7 Dimension; General Electric Co, Cincinnati, $\mathrm{OH}$ ) was operated at $1.6 \mathrm{MHz}$ in the octave mode. The probe was clamped in the water bath with the surface of the right kidney located $3.8 \mathrm{~cm}$ from the transducer face in the 8-cm-deep image. At this position with a 5 -cm focus, the PRPA was maximal, and the $-6 \mathrm{~dB}$ scan plane thickness (beam width) was $4.7 \mathrm{~mm}$. The PRPA at the kidney was determined by calibrated hydrophone measurement (HMA 0200; Onda, Sunnyvale, CA). The pulse duration was 1.5 microseconds with a pulse repetition period of 243 microseconds, which were very similar to the parameters for the GE Vingmed System 5 with an FPA2.5 probe used previously. ${ }^{10}$ The attenuation was previously found to be about $5 \%$ at this low frequency, ${ }^{10}$ and the water PRPA values were reduced by $5 \%$ to approximate the in situ values. Exposures were performed at power settings of $0,-3$, and $-6 \mathrm{~dB}$, with exposure parameters listed in Table 1 . Due to the water path to the rat, the calculated value of $\mathrm{PRPA} / \mathrm{f}^{1 / 2}=1.8$ was larger than the onscreen mechanical index $=1.3$, but was nevertheless within the Food and Drug Administration 510k guideline of mechanical index $=1.9$. The image frames, which were initially at 56.3 frames per second for aiming, were time triggered each second during exposure to allow refill of the tissue with gas bodies after destruction during the imaging scan.

Table 1. Exposure Parameters for the 3 Exposure Power Settings

\begin{tabular}{lcccc}
\hline $\begin{array}{l}\text { Power } \\
\text { Setting, dB }\end{array}$ & $\begin{array}{c}\text { PRPA, } \\
\text { MPa }\end{array}$ & $\begin{array}{c}\text { PRPA/f } \\
\text { MPa/2/2 }\end{array} \mathbf{M H z}^{1 / 2}$ & \multicolumn{2}{c}{ On-Screen Indices } \\
\hline 0 & 2.3 & 1.8 & 1.3 & 0.6 \\
-3 & 1.6 & 1.3 & 0.9 & 0.3 \\
-6 & 1.1 & 0.87 & 0.6 & 0.1 \\
\hline
\end{tabular}

The measured PRPAs were reduced by $5 \%$ to approximate the in situ exposure. The on-screen exposure indices of the mechanical index (MI) and thermal index (TI) are given for comparison and differ substantially from the experimental values because the exposure included a water path of about $3.5 \mathrm{~cm}$.

\section{Ultrasound Contrast Agent}

The US contrast agent was a laboratory replacement for Definity (perflutren lipid microsphere injectable suspension; Lantheus Medical Imaging, Inc, North Billerica, MA), which is no longer sold for research purposes. A replacement contrast agent for Definity was created by using a formula set as close as possible to the Definity formula, as described previously. ${ }^{21} \mathrm{~A}$ lipid blend was made by using powder forms of dipalmitoyl-sn-glycero-3-phosphate (Sigma-Aldrich, Milwaukee, WI), dipalmitoyl-sn-glycero3-phosphocholine (Avanti Polar Lipids, Alabaster, AL), and dipalmitoyl-sn-glycero-3-phosphoethanolamine- $\mathrm{N}$ [methoxy(polyethylene glycol)-5000] (Avanti Polar Lipids). To create the contrast medium, the lipids were dissolved in a mixture of $80 \%$ ( $\mathrm{vol} / \mathrm{vol}$ ) phosphate-buffered saline, $10 \%$ (vol/vol) propylene glycol, and $10 \%$ (vol/vol) glycerol at concentrations of $0.045,0.4$, and $0.3 \mathrm{mg} / \mathrm{mL}$ for dipalmitoyl-sn-glycero-3-phosphate, dipalmitoyl-snglycero-3-phosphocholine, and dipalmitoyl-sn-glycero-3phosphoethanolamine- $N$-[methoxy(polyethylene glycol)5000], respectively. This mixture was heated to $70^{\circ} \mathrm{C}$ to solubilize the lipids and then sterile filtered with a $0.2-\mu \mathrm{m}$ filter. The contrast medium was then aliquoted into used Definity vials that were cleaned and autoclaved. The headspace for each vial was filled with octafluoropropane (HC218, PurityPlus; Metro Welding Supply, Detroit, MI), and the vial was then subsequently capped and sealed.

Before use, the vials were shaken for 45 seconds in a VialMix (Dupont Pharmaceuticals Co, Billerica, MA) exactly as performed for Definity to produce the suspensions of stabilized microbubbles. The replacement contrast agent was compared to actual Definity to gauge its interchangeability with the clinical agent. The concentration and size distribution were measured with a Multisizer 4 (Beckman Coulter, Inc, Indianapolis, IN), which counted microbubbles from 1 to $30 \mu \mathrm{m}$ in diameter. The stability of the two agents was compared by rechecking the suspensions at 5 and 24 hours after mixing. For infusion, the replacement agent was diluted by adding $60 \mu \mathrm{L}$ of the prepared agent to $3 \mathrm{~mL}$ of sterile saline. The dilution was infused at 0.5 $\mathrm{mL} / \mathrm{kg} / \mathrm{min}$ to deliver a $10-\mu \mathrm{L} / \mathrm{kg} / \mathrm{min}$ dose of the agent, which replicated the procedure for previous research. ${ }^{8}$ For exposure, the contrast agent infusion was started with the US on and timed for 1 minutes after the contrast enhancement began to appear in the image, with scanning continuing for 30 seconds after the infusion was stopped. For sham exposure, the rat was scanned for 2 minutes using the maximum $(0 \mathrm{~dB})$ setting, and then the contrast agent was infused for 1 minute with the US off. 


\section{Measured End Points}

The rats were weighed, and physiologic data were collected from most rats for rectal temperature and for heart rate and percent oxygen saturation with a pulse oximeter probe on a hind paw (SurgiVet V3395 TPR; Smiths Medical, Inc, Saint Paul, MN). In addition, the relative urine production was measured for 3 rats anesthetized each with ketamine only or ketamine plus xylazine. This procedure was repeated after 1 week with the anesthetic switched for the rats. The urine output was measured for about 1 hour during the period of deepest anesthesia by placing the rats on a warming pad (T/Pump model TP-500; Gaymar Industries, Inc, Orchard Park, NY) with filter paper circles (Whatman No. 1 qualitative 11-cm circles; Fisher Scientific, Waltham, MA) placed to absorb the urine. The papers were changed as needed, with up to 15 papers needed for the ketamineplus-xylazine group. The difference in the filter paper weights before and after wetting gave the weight of urine produced.

Glomerular capillary hemorrhage was evaluated by histologic analysis as described previously. ${ }^{8}$ Kidney samples were removed, trimmed, and immersion fixed in neutral buffered formalin. Histologic processing was performed at the Research Histology and Immunoperoxidase Laboratory of the University of Michigan Comprehensive Cancer Center Tissue Core. Glomeruli were examined on a slide from the center of the scan plane, and the percentage with any evidence of glomerular capillary hemorrhage, which included as few as 2 erythrocytes in different areas identifiable as Bowman's space, was calculated. In addition, for near-threshold exposures, the number of petechiae was counted on the surface of the kidney as a surrogate for glomerular capillary hemorrhage. The petechiae count is a more sensitive test for glomerular capillary hemorrhage than the histologic sectioning because the blood-filled tubules seen as petechiae arise from glomeruli in a much larger volume of cortex than can be seen in a histologic cross section.

\section{Experimental Plan and Statistics}

For the US exposure experiment, there were a total of 8 separate groups of rats with different exposure parameters. For each anesthetic technique, a sham and exposure at power settings of $0,-3$, and $-6 \mathrm{~dB}$ were performed. The numerical results are presented as the mean \pm standard deviation for groups of 5 samples or plotted as the mean with standard error bars. One rat was lost from the study because of a faulty procedure. For statistical analysis, Student $t$ tests were used to compare means of the measured parameters, with statistical significance assumed at $P=.05$. A 2-way analysis of variance test was used to assess the relative importance of the PRPA and anesthetic method. In addition, the proportion of occurrence was assessed for statistical significance by the $Z$ test (SigmaPlot 11; Systat Software, Inc, San Jose, CA).

\section{Results}

The replacement Definity was compared to the clinical product, with 4 vials of each tested for size and concentration. Table 2 gives the results for measurements immediately after mixing, after 5 hours, and after 24 hours. The differences in the measurements were mostly not statistically significant, except that the diameters of the replacement agent were slightly larger. The size distributions for both agents are shown in Figure 1, as measured soon after mixing. The small differences in size were not expected to result in any important difference in overall US response or glomerular capillary hemorrhage occurrence for the two agents.

The results for the physiologic measurements of the rats are presented in Table 3. The rats all weighed about the same. However, the other parameters for ketamineonly and ketamine-plus-xylazine anesthesia were significantly different. The differences resulted from the moderate sedation caused by the xylazine. Results for the diuresis test for ketamine only with an anesthesia period of $53 \pm 3$ minutes were $0.04 \pm 0.3 \mathrm{~g} / \mathrm{min}$ and a total of $2.1 \pm 1.4 \mathrm{~g}$. For ketamine plus xylazine with a duration of $76 \pm 6$ minutes, the urination rate was $0.13 \pm 0.014 \mathrm{~g} / \mathrm{min}$ and a

Table 2. Comparison of the Replacement and Clinical Definity $(n=4)$ for 3 Time Points

\begin{tabular}{|c|c|c|c|c|c|c|}
\hline \multirow[b]{2}{*}{ Agent } & \multicolumn{2}{|c|}{ Immediate } & \multicolumn{2}{|r|}{$5 \mathrm{~h}$} & \multicolumn{2}{|r|}{$24 \mathrm{~h}$} \\
\hline & $\begin{array}{c}\text { Diameter, } \\
\mu \mathrm{m}\end{array}$ & $\begin{array}{c}\text { Concentration, } \\
10^{9} \mathrm{~mL}^{-1}\end{array}$ & $\begin{array}{c}\text { Diameter, } \\
\mu \mathrm{m}\end{array}$ & $\begin{array}{c}\text { Concentration, } \\
10^{9} \mathrm{~mL}^{-1}\end{array}$ & $\begin{array}{l}\text { Diameter, } \\
\mu \mathrm{m}\end{array}$ & $\begin{array}{c}\text { Concentration, } \\
10^{9} \mathrm{~mL}^{-1}\end{array}$ \\
\hline Definity & $1.6 \pm 0.04$ & $3.4 \pm 0.3$ & $1.8 \pm 0.06$ & $3.4 \pm 0.5$ & $1.8 \pm 0.05$ & $2.9 \pm 0.5$ \\
\hline Replacement & $1.8 \pm 0.11$ & $3.7 \pm 0.3$ & $1.9 \pm 0.06$ & $3.6 \pm 0.23$ & $1.9 \pm 0.02$ & $3.4 \pm 0.2$ \\
\hline$t$ test $P$ & .01 & NS & NS & NS & .01 & NS \\
\hline
\end{tabular}

Data are presented as mean \pm SD. The mean diameter was greater than the 1 - to $30-\mu$ m range. NS indicates not significant $(P>.05)$. 
total of $9.9 \pm 0.8 \mathrm{~g}$. The diuresis caused by the xylazine gave a urine output during anesthesia nearly 5 times that without xylazine $(P<.001)$.

When the contrast agent infusion was begun, the US image showed a relatively dark kidney that brightened within about 15 seconds as the agent entered the kidney circulation, as shown in Figure 2. The central portion of the kidney remained hypoechoic, reflecting the avascular nature of the medulla relative to the high perfusion of the cortex. There was no consistent difference between ketamine-only or ketamine-plus-xylazine anesthesia in the US images. Examples of kidneys exposed at $0 \mathrm{~dB}$ for the two anesthetics are shown in Figure 3.

The glomerular capillary hemorrhage was evident from the appearance of petechiae on the kidney surface, which were blood-filled urinary tubules near the surface. In the histologic analysis, the hemorrhage was apparent as erythrocytes in Bowman's space, as shown in Figure 4.

Figure 1. Plots of microbubble diameter distributions as percentages for 4 vials each of the clinical and replacement contrast agents. There was a small deviation in the small sizes of the replacement agent.

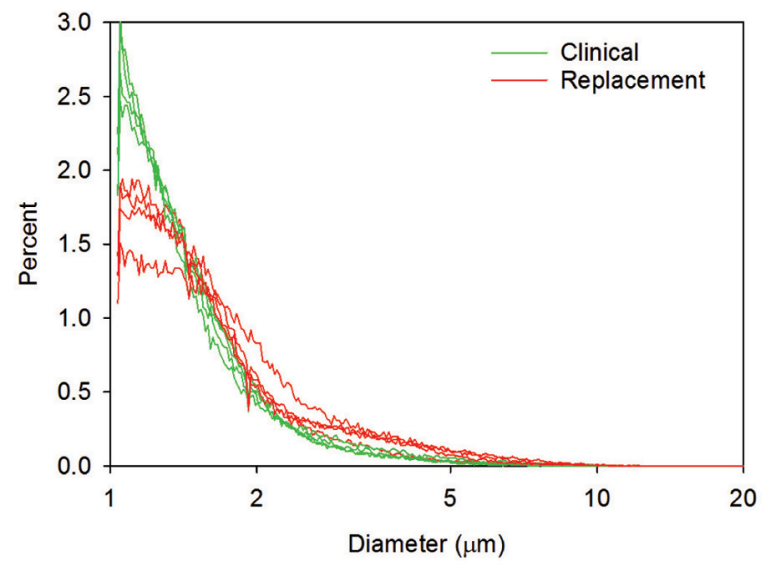

Table 3. Results for the Physiologic Measurements of the Rats at the Time of US Exposure

\begin{tabular}{|c|c|c|c|c|c|}
\hline \multirow[b]{2}{*}{ Test } & \multicolumn{2}{|c|}{ Ketamine Only } & \multicolumn{2}{|c|}{$\begin{array}{c}\text { Ketamine + } \\
\text { Xylazine }\end{array}$} & \multirow{2}{*}{$\begin{array}{c}t \text { test } \\
P\end{array}$} \\
\hline & $n$ & mean \pm SD & $\mathrm{n}$ & mean \pm SD & \\
\hline Weight, g & 21 & $319 \pm 25$ & 20 & $317 \pm 29$ & NS \\
\hline HR, beats/min & 13 & $348 \pm 7$ & 14 & $308 \pm 23$ & $<.01$ \\
\hline $\mathrm{SPO}_{2}, \%$ & 12 & $91 \pm 2$ & 14 & $85 \pm 2$ & $<.01$ \\
\hline Temperature, ${ }^{\circ} \mathrm{C}$ & 15 & $38.2 \pm 0.5$ & 14 & $37.7 \pm 0.5$ & $<.02$ \\
\hline
\end{tabular}

The numbers of rats measured varied because the physiologic tests were not performed on the first 2 to 3 rats of each group, but the results were representative of the entire study. HR indicates heart rate; NS, not significant ( $P>.05$ ); and $\mathrm{SPO}_{2}$, hemoglobin oxygen saturation.
The glomerulus with hemorrhage presumably connects to the convoluted proximal tubules, which are filled with blood and come close to the surface of the kidney. There was no consistent difference in glomeruli or the tubular appearance between ketamine-only and ketamine-plus-xylazine anesthesia in the histologic sections, aside from changes in the number of affected glomeruli.

The results for the percentage of glomerular capillary hemorrhage found in histologic sections are displayed in Figure 5. There was a difference between the magnitude of the hemorrhage between the two anesthetics: in the 2-way analysis of variance, there was a small but significant interaction between the PRPA and the anesthetic $(P=.014)$. Detailed significance tests are listed in Table 4. For exposure at 0 and $-3 \mathrm{~dB}$, the hemorrhage was highly significantly different from that of the shams, and the results for the different anesthetics were also significantly different. At $-6 \mathrm{~dB}$, the histologically determined hemorrhage was not significantly different from that of the shams. However, the more sensitive measure of surface petechiae was significantly greater than that of the shams for both anesthetics. The threshold for glomerular capillary hemorrhage is therefore somewhat lower than the -6-dB level. Assuming that no glomerular capillary hemorrhage would be found at

Figure 2. Example of the contrast enhancement of the rat kidney before (top) and after (bottom) infusion during the intermittent imaging. The bright object to the left of the kidney is bowel, and other bright regions are fat. There was no apparent difference in the appearance of the enhancement for the different anesthetics.
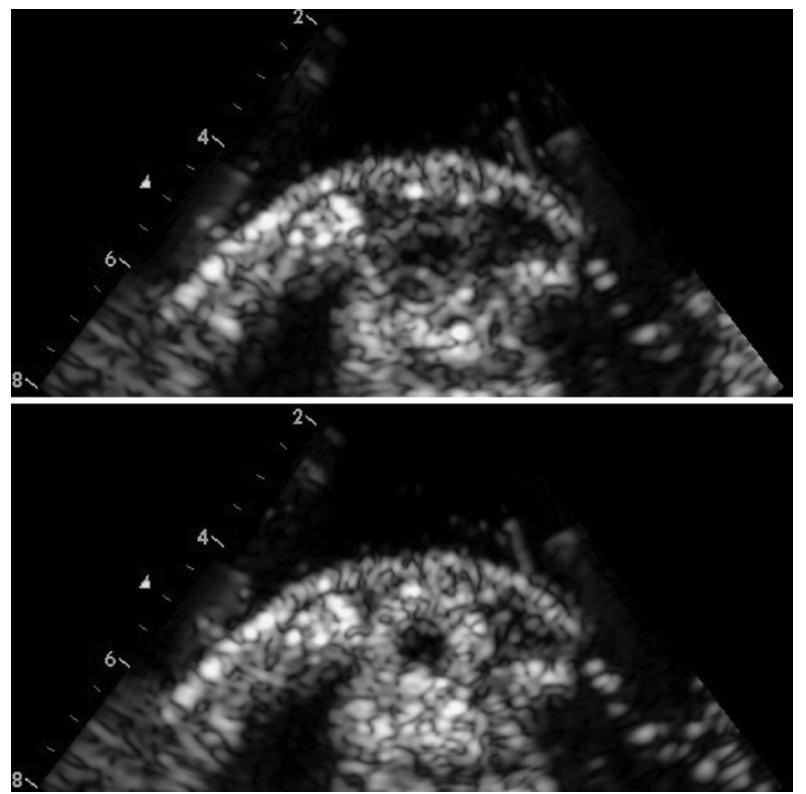
the next lower level $(-8 \mathrm{~dB})$, the threshold was approximated by the mean of these levels, which was $0.94 \mathrm{MPa}$ for both anesthetics. Alternatively, the thresholds can also be estimated by linear regression on the glomerular capillary hemorrhage data, as shown in Figure 5. The linear regression gave coefficients of determination of $r^{2}=0.75$ for ketamine only and $r^{2}=0.95$ for ketamine plus xylazine; the PRPA intercept for both regressions was 1.0 MPa. These results show that the thresholds for glomerular capillary hemorrhage are essentially the same for the two anesthetic methods tested.

\section{Discussion}

The purpose of this research was to test the potential influence of anesthetic methods on the glomerular capillary hemorrhage effect of contrast-enhanced diagnostic US. The recommended anesthetic for research in rats is the combination of ketamine plus xylazine. Xylazine has considerable physiologic effects, which can lead to pulmonary edema ${ }^{17,18}$ and substantial diuresis. ${ }^{19,20}$ Previously, we had found a strong influence of the added xylazine on pulmonary capillary hemorrhage induced by diagnostic US, with a threshold much lower than for ketamine only. ${ }^{15,16}$ In this study, the added xylazine was shown to affect cardiovascular parameters (see Table 3) and to induce substantial diuresis using the normal dosage $(9 \mathrm{mg} / \mathrm{kg})$ in 6 rats, with measured urination rates of $0.04 \pm 0.3 \mathrm{~g} / \mathrm{min}$ using ketamine only and $0.13 \pm 0.014 \mathrm{~g} / \mathrm{min}$ using ketamine plus xylazine. However, these physiologic changes induced by xylazine did not have a large impact on the induction of glomerular capillary hemorrhage by contrast-enhanced diagnostic US. The exposure-response results for the percentage of hemorrhage found in histologic sections is displayed in Figure 5. There was a significant influence of xylazine use on the magnitude of glomerular capillary hemorrhage at $0 \mathrm{~dB}$ : hemorrhage for ketamine only was $45.6 \%$ but increased to $63.2 \%$ for ketamine plus xylazine, an increase of $38 \%(P<.001)$. However, the thresholds for the two anesthetic methods were virtually identical. As indicated by the intercept of linear regressions on the data, the threshold was 1.0 MPa for both, as shown in Figure 5.

Previous research on capillary bioeffects of contrastenhanced diagnostic US has involved several different anesthetic methods. Anesthesia not including xylazine has been common for kidney research, possibly because of concern for the diuretic effect. Wible et $\mathrm{al}^{7}$ used thiobutabarbital sodium for anesthesia, which had an effect on cardiovascular parameters, leading to exclusion of some animals due to hypotension. Thresholds were not specified in that study, but the data indicated zero effect at a mechanical index of approximately 0.8 at $1.8 \mathrm{MHz}$, which corresponded to about a 1.26-MPa PRPA in water. Pentobarbital was used for anesthesia in our initial research,

Figure 3. Photomicrographs of kidneys scanned at $0 \mathrm{~dB}$ during contrast agent infusion for anesthesia with ketamine plus xylazine $(K X)$ and ketamine only (KO). Scale bar indicates $5 \mathrm{~mm}$.
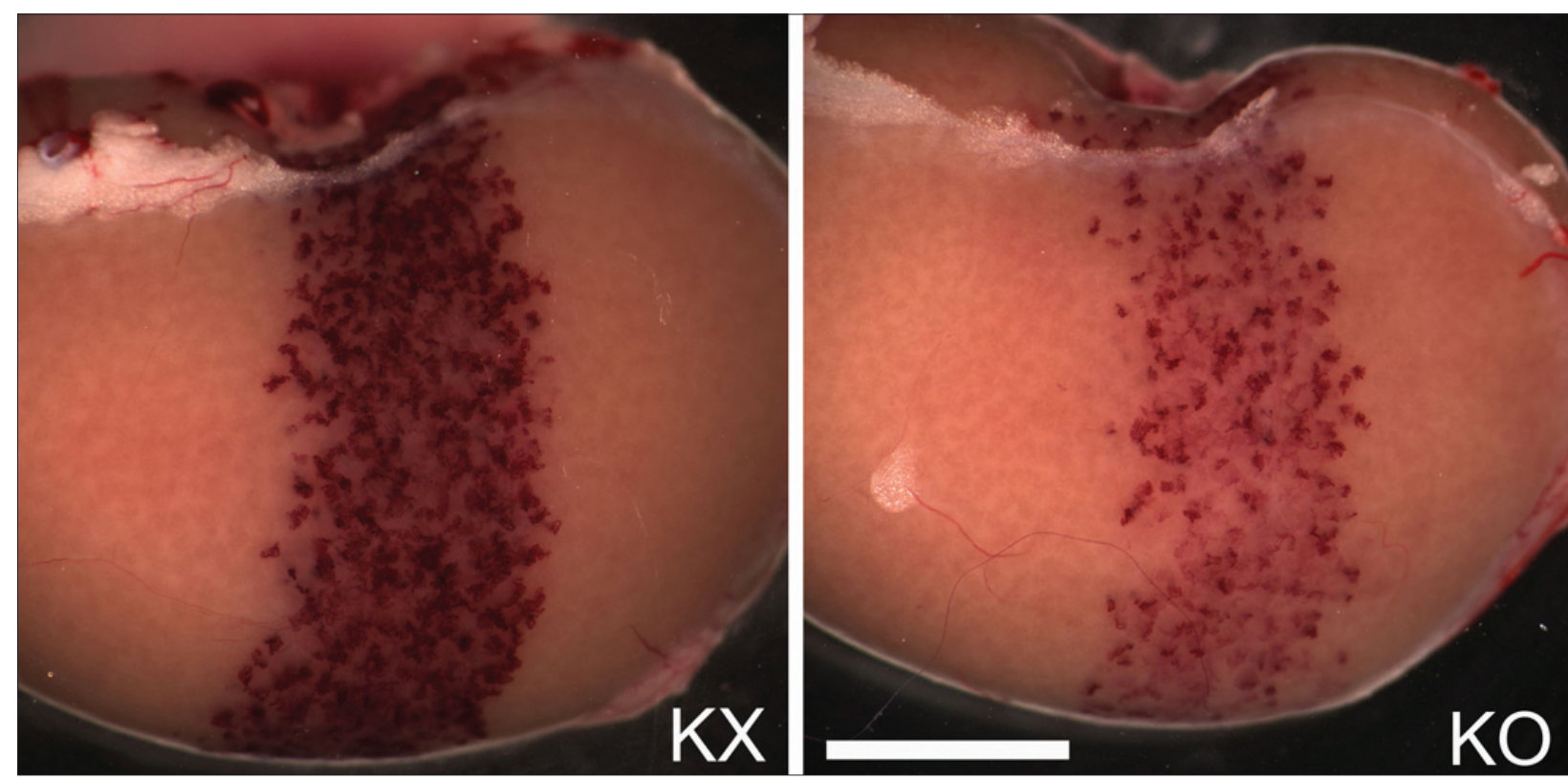
and a threshold of $0.73 \mathrm{MPa}$, including attenuation by a tissue-mimicking phantom, was found for 1.5-MHz diagnostic scanning. ${ }^{8}$ Ketamine plus xylazine also was tested in that study, with no significant difference in results from the pentobarbital anesthesia. In this study, the threshold from linear regression (Figure 5) was $1.0 \mathrm{MPa}$ at $1.6 \mathrm{MHz}$; that was somewhat higher than reported in Miller et al. ${ }^{8}$ In the earlier study, a different method was used to estimate the location of the threshold as midway between the lowest level with a significant effect and the next lower level. If the previous method were applied to this study, the next lower level ( $3 \mathrm{~dB}$ lower) would be $0.77 \mathrm{MPa}$, and the threshold

Figure 4. Photomicrograph of a histologic slide with hematoxylin-eosin staining showing a glomerulus with blood in Bowman's space $(G)$ and the associated blood-containing tubules $(T)$ that appear as petechiae on the surface of the kidney (shown in Figure 3). There was no discernible difference in the histologic appearance of the tissue for the different anesthetics. Scale bar indicates $200 \mu \mathrm{m}$.

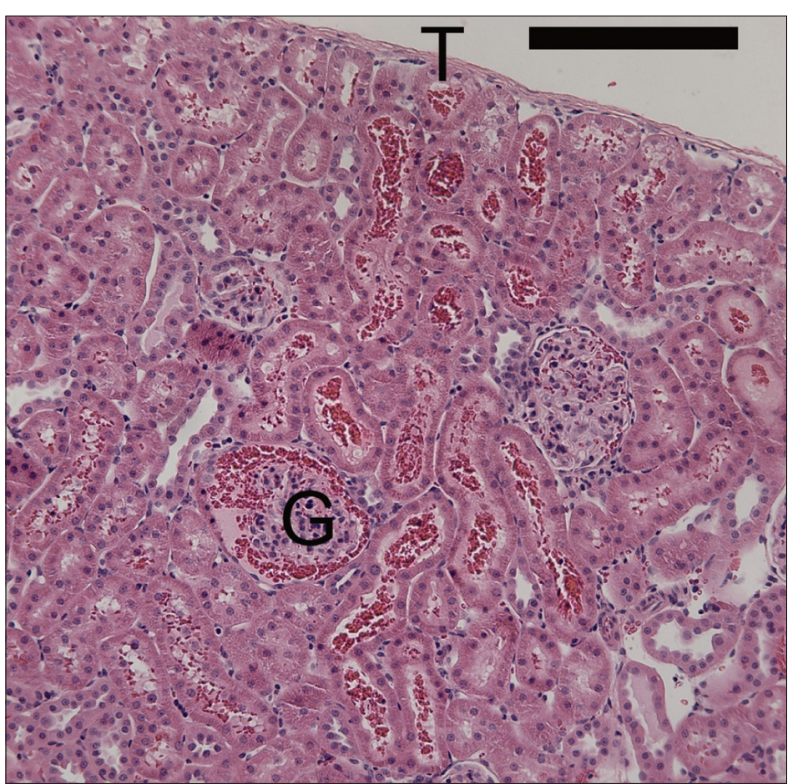

would be $0.94 \mathrm{MPa}$. Adjusting for the frequency dependence (1.6 MHz compared to $1.5 \mathrm{MHz}$ ) gives an adjusted threshold of $0.88 \mathrm{MPa}$ for comparison to the previous threshold of $0.76 \mathrm{MPa}^{8}{ }^{8}$ This difference can be attributed to the normal range of uncertainty in this research, given the different measurement methods and hydrophones used in the two studies.

Anesthesia with ketamine plus xylazine was used in subsequent kidney research, for example, Miller et al,, 912 and in cardiac studies, for example, Miller et $\mathrm{al}^{22}{ }^{22}$ because it was the recommended anesthetic method for rats. In a porcine model of human contrast-enhanced diagnostic US in the kidney, the initial anesthetic was $6-\mathrm{mg} / \mathrm{kg}$ tiletamine/zolazepam plus $2.2 \mathrm{-mg} / \mathrm{kg}$ xylazine, which

Figure 5. The results for glomerular capillary hemorrhage as a percentage of all glomeruli in a histologic section from the center of the scan plane. The bioeffect increases rapidly above thresholds that are indicated by the intercepts of the linear regressions. Although the percentages are different for the higher exposures, the thresholds appear to be nearly identical.

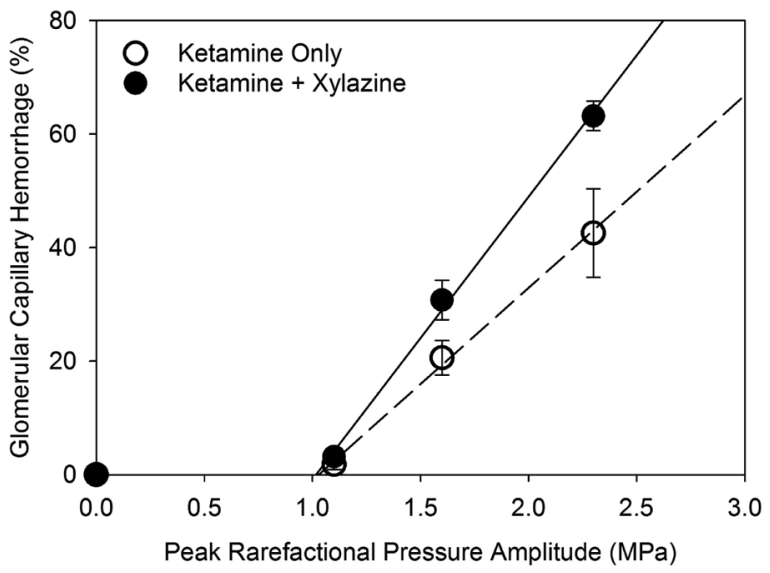

Table 4. Results for Glomerular Capillary Hemorrhage in the $4 \mathrm{KO}$ Groups and 4 KX Groups, Measured by Histologic Analysis as a Percentage of Glomeruli With Hemorrhage in a Histologic Section or as the Number of Petechiae Counted on the Kidney Surface

\begin{tabular}{|c|c|c|c|c|c|}
\hline Group: Measure & KO & $P$ vs Shama & $\mathrm{KX}$ & $P$ vs Sham ${ }^{a}$ & $P K O$ vs KXa \\
\hline $0 \mathrm{~dB}: \% \mathrm{GCH}$ & $45.6 \pm 12.1$ & $<.001$ & $63.2 \pm 5.8$ & $<.001$ & $<.001$ \\
\hline$-3 \mathrm{~dB}: \% \mathrm{GCH}$ & $20.6 \pm 6.8$ & $<.001$ & $30.8 \pm 7.8$ & $<.001$ & .039 \\
\hline$-6 \mathrm{~dB}: \% \mathrm{GCH}$ & $1.8 \pm 2.2$ & .69 (NS) & $3.2 \pm 3.0$ & $.51(\mathrm{NS})$ & .76 (NS) \\
\hline Sham: \% GCH & 0 & NA & 0 & NA & NA \\
\hline$-6 \mathrm{~dB}$ : petechiae & $19.7 \pm 11.0$ & $<.01$ & $23.6 \pm 13.2$ & $<.01$ & .60 (NS) \\
\hline Sham: petechiae & 0 & NA & 0 & NA & NA \\
\hline
\end{tabular}

Data are presented as mean \pm SD. GCH indicates glomerular capillary hemorrhage; KO, ketamine only; KX, ketamine plus xylazine; NA, not applicable; and NS, not significant.

aTwo-way analysis of variance. 
was supplemented for maintenance with $1 \%$ to $3 \%$ isoflurane in oxygen. ${ }^{9}$ No threshold was determined in that study, but $31.7 \%$ glomerular capillary hemorrhage was found for transabdominal scanning at $1.5 \mathrm{MHz}$ with a $1.7-\mathrm{MPa}$ PRPA in situ, which was consistent with results in rats.

Overall, these results suggest that anesthetic methods in research can influence the magnitude of capillary bioeffects of contrast-enhanced diagnostic US, but that thresholds are approximately the same for different anesthetic methods. These findings are in contrast to the pronounced influence of anesthetic methods on the induction of pulmonary capillary hemorrhage by diagnostic US. ${ }^{16}$ This difference may result from the physical mechanism of contrast-enhanced diagnostic US bioeffects. The interaction of diagnostic US pulses with contrast agent microbubbles generates a local high-amplitude secondary source of capillary perturbation when cavitation nucleation occurs, a phenomenon that is likely dominated by local physical conditions, with little influence by physiologic parameters.

\section{References}

1. Averkiou M, Powers J, Skyba D, Bruce M, Jensen S. Ultrasound contrast imaging research. Ultrasound Q2003; 19:27-37.

2. Malhi H, GrantEG, DuddalwarV. Contrast-enhanced ultrasound of the liver and kidney. Radiol Clin North Am 2014; 52:1177-1190.

3. Granata A, Zanoli L, Insalaco M, et al. Contrast-enhanced ultrasound (CEUS) in nephrology: Has the time come for its widespread use? Clin Exp Nephrol 2015; 19:606-615.

4. Riccabona M, Avni FE, Damasio MB, et al. ESPR Uroradiology Task Force and ESUR Paediatric Working Group_-imaging recommendations in paediatric uroradiology, part $\mathrm{V}$ : childhood cystic kidney disease, childhood renal transplantation and contrast-enhanced ultrasonography in children. Pediatr Radiol 2012; 42:1275-1283.

5. Piscaglia F, Nolsøe C, Dietrich CF, et al. The EFSUMB Guidelines and Recommendations on the Clinical Practice of Contrast Enhanced Ultrasound (CEUS): update 2011 on non-hepatic applications. Ultraschall Med 2012; 33:33-59.

6. Miller DL, Averkiou MA, Brayman AA, et al. Bioeffects considerations for diagnostic ultrasound contrast agents. JUltrasound Med2008; 27:611-632.

7. Wible JH Jr, Galen KP, Wojdyla JK, Hughes MS, Klibanov AL, Brandenburger GH. Microbubbles induce renal hemorrhage when exposed to diagnostic ultrasound in anesthetized rats. Ultrasound Med Biol 2002; 28:1535-1546.

8. Miller DL, Dou C, Wiggins RC, Wharram BL, Goyal M, Williams AR. An in vivo rat model simulating imaging of human kidney by diagnostic ultrasound with gas-body contrast agent. Ultrasound Med Biol 2007; 33:129-135.

9. Miller DL, Dou C, Wiggins RC. Contrast-enhanced diagnostic ultrasound causes renal tissue damage in a porcine model. J Ultrasound Med 2010; 29:1391-1401.
10. Miller DL, Dou C,Wiggins RC. Frequency dependence of kidney injury induced by contrast-aided diagnostic ultrasound in rats. Ultrasound Med Biol 2008; 34:1678-1687.

11. Williams AR, Wiggins RC, Wharram BL, et al. Nephron injury induced by diagnostic ultrasound imaging at high mechanical index with gas body contrast agent. Ultrasound Med Biol 2007; 33:1336-1344.

12. Miller DL, Dou C, Wiggins RC. Glomerular capillary hemorrhage induced in rats by diagnostic ultrasound with gas-body contrast agent produces intratubular obstruction. Ultrasound Med Biol 2009; 35:869877.

13. Child SZ, Hartman CL, Schery LA, Carstensen EL. Lung damage from exposure to pulsed ultrasound. Ultrasound Med Biol 1990; 16:817-825.

14. Church CC, Carstensen EL, Nyborg WL, Carson PL, Frizzell LA, Bailey MR. The risk of exposure to diagnostic ultrasound in postnatal subjects: nonthermal mechanisms. J Ultrasound Med 2008; 27:565-592.

15. Miller DL, Suresh MV, Dou C, Yu B, Raghavendran K. Characterization of ultrasound-induced pulmonary capillary hemorrhage in rats. Microvasc Res 2014; 93:42-45.

16. Miller DL, Dou C, Raghavendran K. Anesthetic techniques influence the induction of pulmonary capillary hemorrhage during diagnostic ultrasound scanning in rats. JUltrasound Med 2015; 34:289-297.

17. Amouzadeh HR, Sangiah S, Qualls CWJr, Cowell RL, Mauromoustakos A. Xylazine-induced pulmonary edema in rats. Toxicol Appl Pharmacol 1991; 108:417-427.

18. Amouzadeh HR, Qualls CW Jr, WyckoffJH III, et al. Biochemical and morphological alterations in xylazine-induced pulmonary edema. Toxicol Pathol 1993; 21:562-571.

19. Cabral AD, Kapusta DR, Kenigs VA, Varner KJ. Central $\boldsymbol{\alpha}_{2}$-receptor mechanisms contribute to enhanced renal responses during ketaminexylazine anesthesia. Am J Physiol 1998; 275:R1867-R1874.

20. Miller JH, McCoy KD, Coleman AS. Renal actions of the a2-adrenoceptor agonist, xylazine, in the anaesthetised rat. NZ Vet J2001; 49:173180.

21. Miller DL, Dou C, Lu X, et al. Use of theranostic strategies in myocardial cavitation-enabled therapy. Ultrasound Med Biol 2015; 41:1865-1875.

22. Miller DL, Dou C,Lucchesi BR. AreECG premature complexes induced by ultrasonic cavitation electrophysiological responses to irreversible cardiomyocyte injury? Ultrasound Med Biol 2011; 37:312-320. 